\title{
MODELLING OF QUANTUM DOT SOLAR CELLS FOR CONCENTRATOR PV APPLICATIONS
}

\author{
A. Ogura, ${ }^{1,2, a)}$ T. Morioka, ${ }^{1,2}$ P. García-Linares, ${ }^{3}$ E. Hernández, ${ }^{3}$ I. Ramiro, ${ }^{3}$ I. Artacho, ${ }^{3}$ E. Antolín, ${ }^{3}$ \\ A. Martí, ${ }^{3}$ A. Luque, ${ }^{3}$ M. Yamaguchi, ${ }^{1,4}$ and Y. Okada ${ }^{1,2}$ \\ ${ }^{1}$ Research Center for Advanced Science and Technology (RCAST), The University of Tokyo, Meguro-ku, Tokyo, Japan \\ ${ }^{2}$ School of Engineering, The University of Tokyo, Meguro-ku, Tokyo, Japan \\ ${ }^{3}$ Instituto de Energía Solar, Universidad Politécnica de Madrid, Madrid, Spain \\ ${ }^{4}$ Toyota Technological Institute, Tempaku, Nagoya, Japan
}

\begin{abstract}
An equivalent circuit model is applied in order to describe the operation characteristics of quantum dot intermediate band solar cells (QD-IBSCs), which accounts for the recombination paths of the intermediate band (IB) through conduction band (CB), the valence band (VB) through IB, and the VB-CB transition. In this work, fitting of the measured dark $J-V$ curves for QD-IBSCs (QD region being non-doped or direct Si-doped to $n$-type) and a reference GaAs $p-i-n$ solar cell (no QDs) were carried out using this model in order to extract the diode parameters. The simulation was then performed using the extracted diode parameters to evaluate solar cell characteristics under concentration. In the case of QDSC with Si-doped (hence partially-filled) QDs, a fast recovery of the open-circuit voltage $\left(V_{o c}\right)$ was observed in a range of low concentration due to the IB effect. Further, at around 100X concentration, Si-doped QDSC could outperform the reference GaAs $p-i$ $n$ solar cell if the current source of IB current source were sixteen times to about $10 \mathrm{~mA} / \mathrm{cm}^{2}$ compared to our present cell.
\end{abstract}

\section{Introduction}

The intermediate band solar cells (IBSCs) [1], which can be realized with a quantum dot (QD) superlattice inserted in the active region, have been proposed as a way to exceed the Shockley-Queisser limit. IBSCs can absorb sub-bandgap photons to generate additional currents by using two-step photoabsorption. One absorption step is from the VB to IB, and another step is from the IB to CB. Several circuit models to account for the two-step optical transitions have been proposed in order to evaluate the characteristics of QDSCs $[2,3]$. The fitting of the dark $J-V$ curves for QDSCs has also been carried out by using a modified circuit model [4]. In this work, the characteristics of QDSCs under concentration were simulated using the extracted diode parameters from the measured dark $J-V$ curves.

\section{Diode equivalent circuit model}

Fig. 1 shows the circuit model used for the simulation in this work. The model consists of a diode $D_{\mathrm{VC}}$ (recombination path from the CB to VB), $D_{\mathrm{IC}}$ (recombination path from the $C B$ to IB), $D_{V I}$ (recombination path from the IB to $V B$ ), and $J L_{V c}$ (current source from the
VB to $C B$ ), $J L_{I C}$ (current source from the IB to CB), $J L_{V I}$ (current source from the VB to IB), and a series resistance $R_{\mathrm{S}}$, and a shunt resistance $R_{\mathrm{sh}}$, respectively. The diode ideality factor $n$ for $D_{v c}$ is a variable $(1 \leqq n \leqq 2)$ for the fitting of experimental data. Here $D_{\mathrm{IC}}$ and $D_{\mathrm{VI}}$ are set to be $n=1$ because the recombination paths via intermediate band are assumed to be (and ideally) due to radiative recombination.

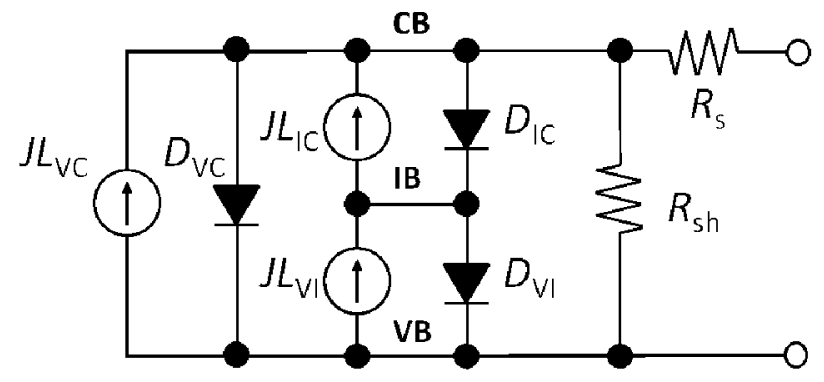

Fig. 1 Diode equivalent circuit model.

The following equations represent the current components passing through each diode,

$$
\begin{aligned}
& J_{V C}=J_{0 V C}\left[\exp \left(\frac{q V_{V C}}{n k T}\right)-1\right] \\
& J_{V I, I C}=J_{0 V, 0 I C}\left[\exp \left(\frac{q V_{V, I C}}{k T}\right)-1\right],
\end{aligned}
$$

where $V_{v c}, V_{V I, I C}$ are the applied voltage across each diode, $J_{0 \mathrm{VC}}, J_{0 \mathrm{~V}}, J_{\text {olc }}$ are the saturation current densities, $k$ is the Boltzmann constant, and $T$ is the absolute temperature, respectively. Thus for this circuit model, six variable fitting parameters, $J_{0 \vee C}, J_{0 \mathrm{VI}}, J_{\mathrm{OIC}}, n, R_{\mathrm{sh}}$, and $R_{\mathrm{S}}$, are considered.

\section{Quantum dot structure}

We have fabricated a reference GaAs $p-i-n$ solar cell, and three types of $p-i-n$ QDSCs. One sample consists of 25 multi-stacked InAs QDs fabricated with direct Si-doping [5], and the other QDSCs are without doping (non-doped) of 25 and 50 multi-stacked InAs QD layers, respectively. The samples were grown by using atomic hydrogen-assisted 
RF-MBE on GaAs (001) substrate (Fig. 2). The 25 or 50 pairs of a 2.0 monolayers (MLs) of InAs QD layer and a 20 $\mathrm{nm}$-thick $\mathrm{GaN}_{0.01} \mathrm{As}_{0.99}$ spacer layer were incorporated into the $i$-layer region as reported previously [5]. The net average lattice strain was minimized by using straincompensation technique, in which GaNAs dilute nitride was used as a strain-compensating layer (SCL) [6].

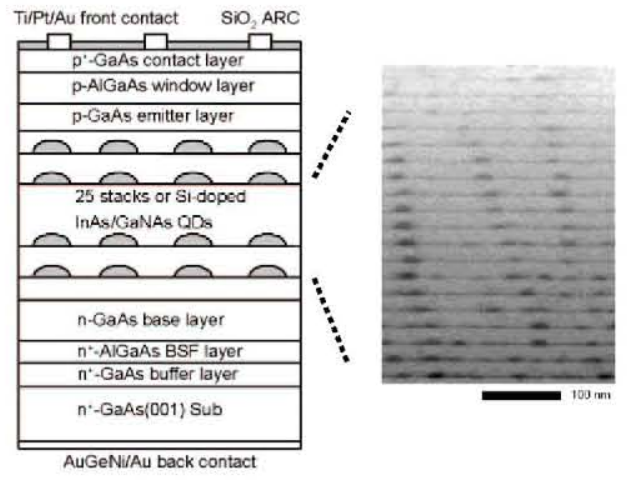

Fig. 2 Schematic layer structure of QDSC and cross sectional STEM image of QD region studied in this work.
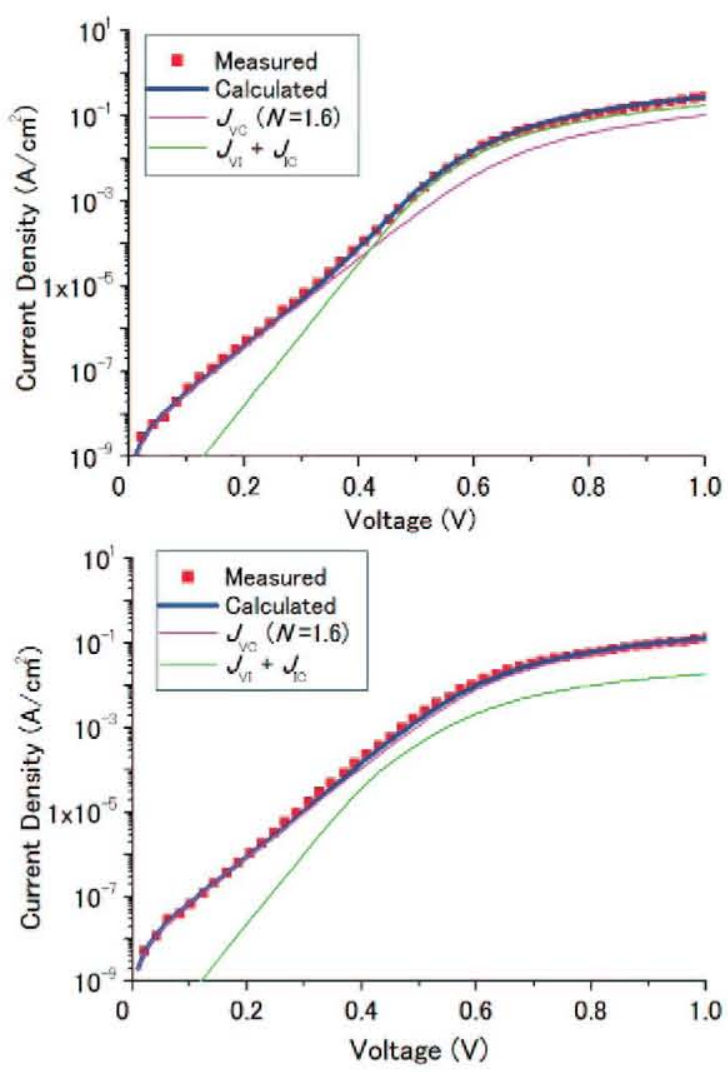

Fig. 3 Fitting of the dark $J-V$ curve for (a) QDSC with 25 direct Si-doped QD stacks, (b) QDSC with 25 nondoped QD stacks.

\section{Results and Discussion}

\section{Fitting of Dark J-V Curves}

Fig. 3(a) and 3(b) show the results of simulated and measured curves for QDSC with 25 direct Si-doped and non-doped QD stacks, respectively, by using the model as shown Fig. 1.The simulation fits well with the experimental data. In this case, the diode ideality factor $n$ of $D_{\mathrm{vc}}$ is equal to $n=1.6$. Because we extracted the diode ideality factor $n$ $=1.6$, when simulating the reference $p-i-n \mathrm{GaAs}$ solar cell by using the model as shown Fig. 1 without the intermediate band diodes, $D_{V I}$ and $D_{I C}$. Other parameters obtained from the fitting are summarized in Table I. While the effect of intermediate band diodes can be clearly seen for the doped QDSC in the bias range $V>\sim 0.4 \mathrm{~V}$, nondoped QDSCs (with 25 and 50 multi-stacked layers) show no apparent or negligibly small contributions from IB diodes.

Table I Circuit parameters extracted by the fitting. ( $n=1.6$ and $n=1$ represent diode ideality factors.)

\begin{tabular}{|l|l|l|l|l|l|}
\hline & $\begin{array}{l}J_{\text {OVC }} \\
(n=1.6) \\
\mathrm{A} / \mathrm{cm}^{2}\end{array}$ & $\begin{array}{l}J_{\text {OIC }} \\
(n=1) \\
\mathrm{A} / \mathrm{cm}^{2}\end{array}$ & $\begin{array}{l}J_{\text {OVI }} \\
(n=1) \\
\mathrm{A} \mathrm{cm}\end{array}$ & $\begin{array}{l}R \mathrm{~s} \\
\Omega \mathrm{cm}^{2}\end{array}$ & $\begin{array}{l}R \mathrm{sh} \\
\Omega \mathrm{cm}^{2}\end{array}$ \\
\hline $\begin{array}{l}\text { GaAs p-i-n } \\
\text { (reference) }\end{array}$ & $9.0 \times 10^{-12}$ & - & - & 0.9 & $1.0 \times 10^{6}$ \\
\hline $\begin{array}{l}\text { QDSC } \\
\text { 25 layers } \\
\text { Non-doped }\end{array}$ & $7.0 \times 10^{-9}$ & $1.0 \times 10^{-4}$ & $1.0 \times 10^{-11}$ & 2.3 & $1.0 \times 10^{6}$ \\
\hline $\begin{array}{l}\text { QDSC } \\
\text { 25 layers } \\
\text { Si-doped }\end{array}$ & $3.0 \times 10^{-9}$ & $4.0 \times 10^{-3}$ & $7.0 \times 10^{-12}$ & 1.0 & $1.0 \times 10^{6}$ \\
\hline $\begin{array}{l}\text { QDSC } \\
\text { 50 layers } \\
\text { Non-doped }\end{array}$ & $1.5 \times 10^{-8}$ & $8.0 \times 10^{-5}$ & $1.0 \times 10^{-11}$ & 0.025 & $1.0 \times 10^{6}$ \\
\hline
\end{tabular}

\section{Simulation of QDSC Characteristics under Concentration}

Using the parameters obtained in the previous section (Table I), simulation of concentration characteristics of $V_{o c}$ and efficiency of QDSCs were carried out. The estimated values of current sources, $J L_{V C}, J L_{V l}, J L_{I C}$, were used, c.f. Ref. [7], and a blackbody radiation at $5800 \mathrm{~K}$ was used as the solar spectrum. Fig. 4 compare the simulated values of $V_{\text {oc }}$ for QDSCs with 25 QD stacks with direct Si-doped and non-doped, respectively. 


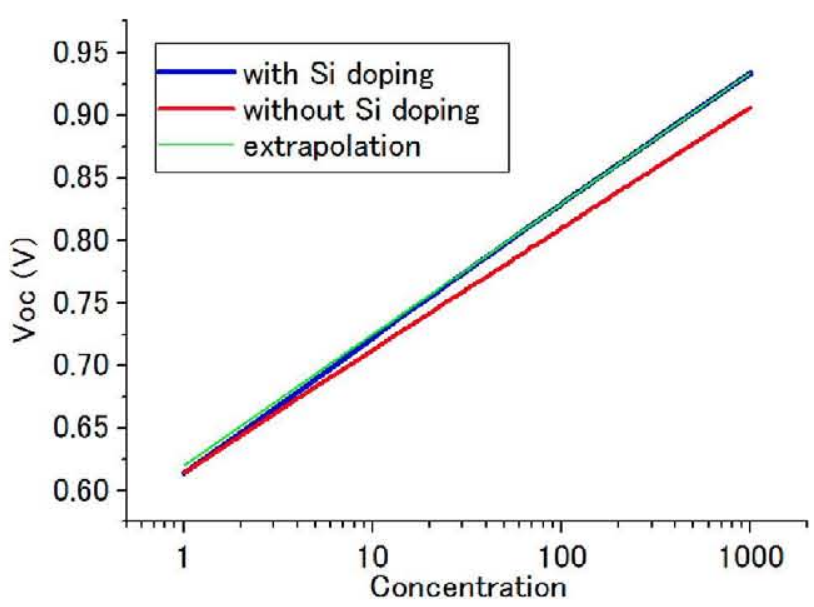

Fig. 4 Simulation of $V_{\text {oc }}$ for QDSCs with 25 QD stacks with direct Si-doped and non-doped, respectively.

As can be seen, the slope of QDSC with direct Si doping is steeper than that for non-doped QDSC. The fitted curve (green in color) represents an extrapolation from the range of 100 and 1000X for QDSC with direct Si doping, and Fig. 5 show the enlarged view of the dependence of $V_{o c}$ on concentration in a low concentration region up to $10 \mathrm{X}$. For QDSC with Si-doped (partially-filled) QDs, a fast recovery of $V_{\text {oc }}$ was observed in this range due to the IB effect. The diode ideality factor $n$ changes from $V=\sim 0.4 \mathrm{~V}$, as can be seen from Fig. 3(a), and this effect acts to recover quickly and increases $V_{\text {oc }}$ more steeply with concentration.

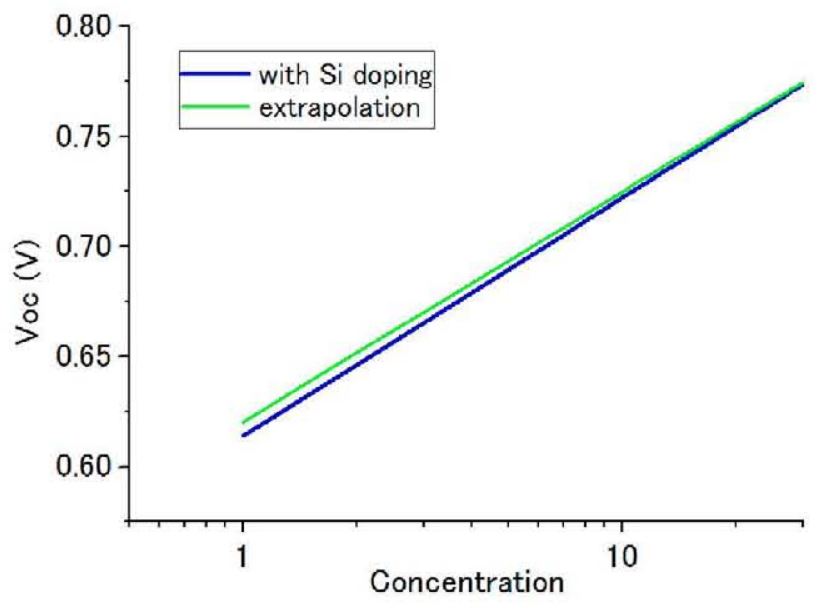

Fig. 5 Simulation of $V_{\text {oc }}$ for QDSC with 25 direct Sidoped QD stacks in the low concentration range.

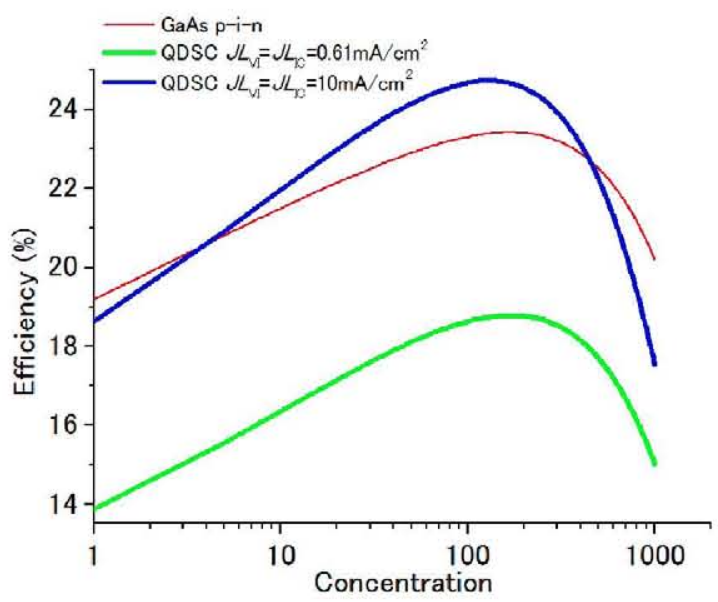

Fig. 6 Simulated efficiencies for QDSC with 25 direct Si-doped QD stacks and reference GaAs $p$-i-n cell.

Lastly, Fig. 6 shows the simulated characteristics of solar cell efficiencies as a function of concentration. The results for the reference GaAs $p-i-n$ solar cell, and QDSC with 25 direct Si-doped QD stacks with different current sources of $0.61 \mathrm{~mA} / \mathrm{cm}^{2}$ and $10 \mathrm{~mA} / \mathrm{cm}^{2}$ are shown. The parameters obtained in the previous section were used except for $R_{\mathrm{s}}$, which was taken to be $0.01 \Omega \mathrm{cm}^{2}$.

The efficiency of QDSC $\left(J L_{V I}=J L_{V I}=0.61 \mathrm{~mA} / \mathrm{cm}^{2}\right)$ fabricated recently in our laboratory [7] is inferior to that of the reference cell over the entire concentration range. However, if the QDSC can be configured to generate a higher current $J L_{V I}=J L_{I C}=10 \mathrm{~mA} / \mathrm{cm}^{2}$, which is about sixteen times the value of our present cell, then efficiency could outperform the $\mathrm{GaAs}$ reference cell at around $100 \mathrm{X}$ concentration.

\section{Summary}

We have successfully simulated the measured dark $J-V$ curves for QD-IBSCs (QD region being non-doped or direct Si-doped to $n$-type) and a reference GaAs $p$ - $i-n$ solar cell (no QDs) using a diode equivalent circuit model. The simulation was then performed using the extracted diode parameters to evaluate the solar cell characteristics under concentration. In the case of QDSC with Si-doped partially-filled QDs, a fast recovery of $V_{\text {oc }}$ was observed in a range of low concentration due to the IB effect. Further, at around 100X concentration, QDSC with Si-doped QDs could outperform the reference GaAs $p-i-n$ solar cell if the current source of IB current source is increased to about $10 \mathrm{~mA} / \mathrm{cm}^{2}$, about sixteen the value of our recently fabricated cell. 


\section{Acknowledgements}

This work has been funded by the Project DENQUIBAND (PLE2009-0045), and also supported by the Incorporated Administrative Agency New Energy and Industrial Technology Development Organization (NEDO) and Ministry of Economy, Trade and Industry (METI), Japan.

A. Ogura would like to acknowledge the support from the Global Center of Excellence (G-COE) "Secure-Life Electronics" sponsored by the Ministry of Education, Culture, Sports, Science and Technology (MEXT), Japan.

\section{References}

[1] A. Luque et al., "Increasing the efficiency of ideal solar cells by photon induced transitions at intermediate levels", Phys. Rev. Lett. 78, 1997, pp. 5014-5017.

[2] A. Luque et al., "General equivalent circuit for intermediate band devices: Potentials, currents, and electroluminescence", J. Appl. Phys. 96, 2004, pp. 903909.

[3] A. Marti et al., "Elements of the design and analysis of quantum-dot intermediate band solar cells", Thin Solid Films 516, 2008, pp. 6716-6722.

[4] A. Luque et al., "Multiple levels in intermediate band solar cells", Appl. Phys. Lett. 96, 2010, pp. 013501013503

[5] T. Morioka et al., "Multi-stacked InAs/GaNAs quantum dots with direct Si doping for use in intermediate band solar cell", 35 ${ }^{\text {th }}$ IEEE PVSC, 2010, pp. 001834-001837.

[6] R. Oshima et al., "Strain-compensated InAs/GaNAs quantum dots for use in high-efficiency solar cells", Appl. Phys. Lett. 93, 2008, pp. 083111-083113

[7] Y. Okada et al., "Increase in photocurrent by optical transitions via intermediate quantum states in directdoped InAs/GaNAs strain-compensated quantum dot solar cell", J. Appl. Phys. 109, 2011, pp. 024301-024304 